\title{
ZONACIÓN DE UN ARROYO DE MONTAÑA (SIERRA DE BÉJAR, ESPAÑA) EN FUNCIÓN DE LA VEGETACIÓN MACROFÍTICA
}

\author{
A. Puerto' ${ }^{1}$ M. Rico², J.A. García' y B.G. de Bikuña ${ }^{3}$ \\ 1. Área de Ecología, Facultad de Biología, Universidad de Salamanca. 37008 Salamanca. España. \\ 2. Instituto de Recursos Naturales y Agrobiología (CSIC). 37008 Salamanca. España. \\ 3. Laboratorio de Ecología, Facultad de Ciencias, Universidad del País Vasco (EHU). 48080 Bilbao. España
}

Palabras clave: mountain stream, zonation, macrophytes, diversity, Spain.

\author{
ABSTRACT \\ ZONATION OF A MOUNTAIN STREAM (BÉJAR SIERRA, SPAIN) \\ AS A FUNCTION OF ITS MACROPHYTIC VEGETATION
}

A study is made of the macrophytic vegetation of the head and banks of a mountain stream («Arroyo del Oso»), situated in the Béjar Sierra (Central-Western Spain).

The results points to a marked zonation both for the multiple alternatives present at the head of the stream and along its course. The soil substrate does not affect this aspect since it is uniform throughout the basin; contrariwise, the shade from nearby trees is of special relevance.

The zonation, above all in the longitudinal sense, is more quantitative than qualitative, as shown by classification analysis. Diversity and heterogeneity are related to the levels of affinity reached in such analysis, though there are ccrtain exceptions which are easy to explain in terms of the number of species and dominance.

\section{INTRODUCCIÓN}

Es bien conocido que las plantas enraizadas constituyen la base de las redes tróficas en aquellos arroyos en que la fuerte corriente arrastra al fitoplancton (WETzEL, 1975); al mismo tiempo, son responsables de las funciones de transporte y subdivisión del espacio (Holmes, 1983), si bien la interpretación se ve dificultada por el gran número de factores ambientales significativos en la distribución de las especies (MARGALEF, 1983).

Los trabajos realizados en arroyos de montaña suelen presentar una cierta limitación, ya que los factores que influyen en la distribución de la vegetación macrofítica es frecuente que queden reducidos a la acción de la luz (Westlake, 1966) o del sustrato (HoFf, 1978), si bien su importancia

Limnetica, 7: 113-122 (1991)

(C) Asociación Española de Limnología, Madrid, Spain es distinta según se trate de plantas del interior del cauce o ribereñas. El contraste es evidente con las comunidades leníticas, cuyas elevadas posibilidades de diferenciación y mayor constancia tipológica garantizan conclusiones más generalizables (WhitTon, 1975).

A pesar de esta panorámica poco prometedora, existen indicios de que es posible sustraerse al simple binomio luz-sustrato. AMOR (1984) indica que, en ocasiones, la vegetación parece distribuirse siguiendo pautas zonales independientes en cierto modo de las características del medio. La pretensión de este trabajo es insistir sobre la presencia de zonaciones que no responden por completo a los dos factores citados. Se trata de un arroyo de montaña, por lo que las condiciones de partida vienen a ser casi las mismas a las que se refieren los comentarios precedentes, si bien su longitud es algo superior a la de los estudiados por otros autores. 


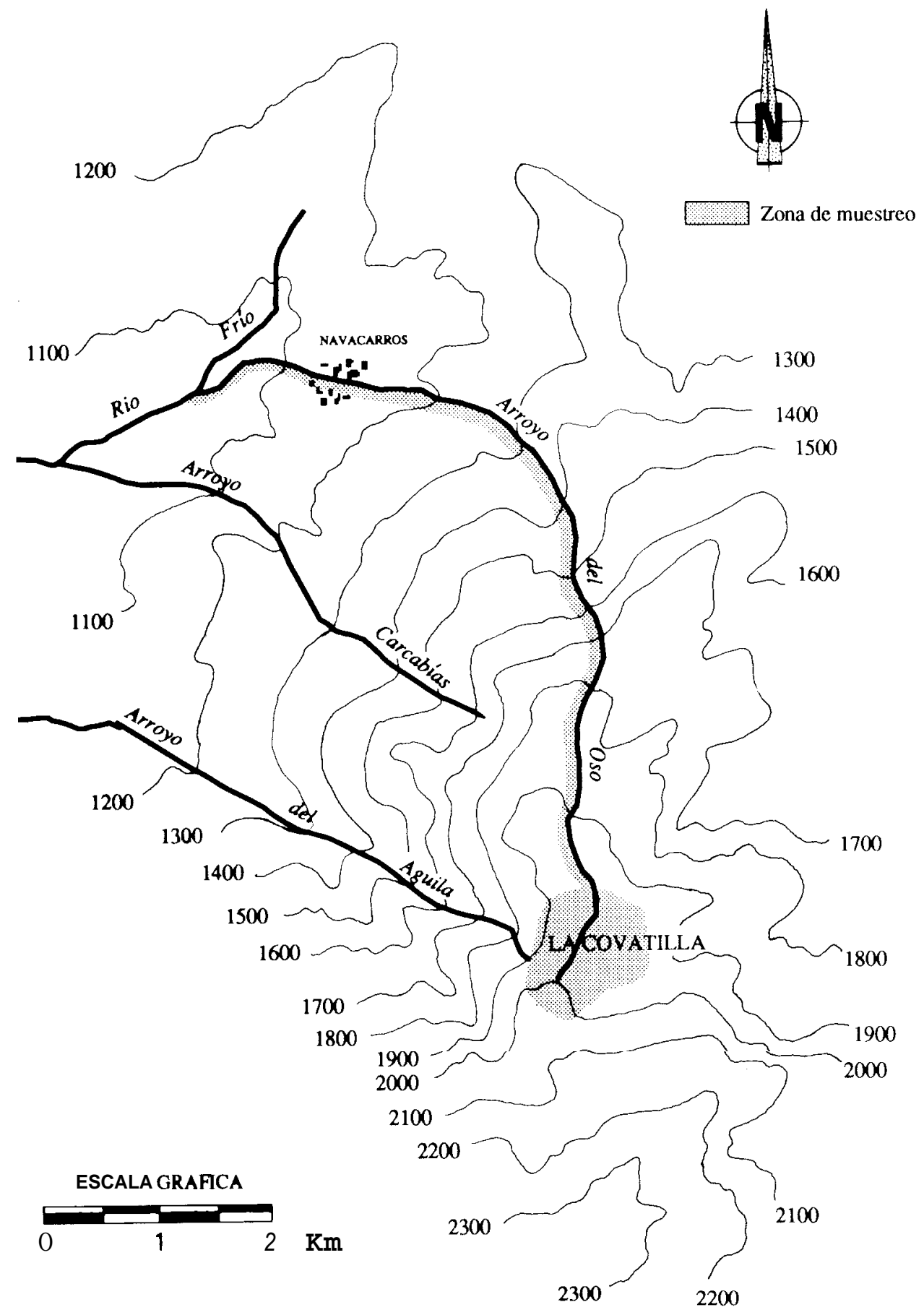

Figura 1.- Esquema topográfico y fluvial, indicando el área de muestreo Topographic and fluvial scheme showing sampling arca. 


\section{MATERIAL Y MÉTODOS}

El Arroyo del Oso, objeto de este estudio, está comprendido en los términos municipales de $\mathrm{Na}$ vacarros y La Hoya (Sierra de Béjar), al sureste de la provincia de Salamanca. Dicha zona está surcada por diversos arroyos de montaña, de acción erosiva remontante, los cuales pertenecen a la cuenca del Tajo. Entre ellos (fig. 1), destaca el Arroyo del Oso, de unos $8 \mathrm{~km}$ de longitud, que nace en La Covatilla a 2000 m.s.n.m. y va a desembocar en el Río Frío, el cual a su vez vierte sus aguas en el Río Cuerpo de Hombre. Todos estos arroyos presentan importantes variaciones de caudal, con un máximo en primavera durante el deshielo y un mínimo en el período estival, en correspondencia con el clima de tipo húmedo, templado-frío y sin aridez (Garmendia, 1964).

Según Garcia Rodríguez (1964) en los enclaves cacuminales predominan los litosoles con afloramientos rocosos, mientras que más abajo los suelos pasan a ser cambisoles dístricos y húmicos. La geología es típicamente granítica, aspecto general que se encuentra en los cauces, constituidos por sustratos duros; predominan los granitos heterogéneode anatexia y las migmatitas, con cordones de granito aplítico de nódulos cortando el territorio ocasionalmente de NE a SW. Todos estos factores actúan como condicionantes de la vegetación, que ha sido descrita por Herrero (1985), y de forma más general por Rivas MARTíNEZ (1975).

En la figura 1 se representa la zona de muestreo. En los enclaves superiores se realizaron 31 inventarios previamente establecidos, tratando de que quedara representada la variabilidad de pozas, charcas, manantiales, comunidades higroturbosas, etc. Otros 79 inventarios fueron dispuestos regularmente a lo largo del arroyo, a intervalos de $100 \mathrm{~m}$, y siempre por su margen izquierda (dado el encajamiento y la posición basal de los muestreos, no parecen existir variaciones aparentes entre ambas márgenes). Para cada muestreo se utilizaron 10 cuadrados de $1 \times 1 \mathrm{~m}$, cinco de los cuales, colindantes y en sentido longitudinal a la orilla $(5 \times 1 \mathrm{~m})$, se situaban en el cauce, y los otros cinco, junto a los anteriores y con la misma disposición, en la orla de inundación de la ribera. Las especies presentes se cuantificaron anotándose la cobertura, entendida como la apreciación vi- sual de su proyección sobre el sustrato; a pesar de que dicha apreciación tiene algo de subjetivo, el hecho de proceder individualmente con cada una de las unidades elementales, calculándose posteriormente la media, mitiga las desviaciones ocasionales que puedan producirse.

El resultado es una matriz (coberturas medias) de 110 inventarios $\times 97$ variables (96 especies, a las que se añaden los musgos como grupo indiferenciado), que fué tratada mediante el conocido análisis factorial de correspondencias, complementado por el de afinidad. Dado que el análisis de correspondencias, en la modalidad utilizada, incorpora una caracterización para cada eje de los distintos inventarios en función de las especies, el establecimiento de grupos se ha basado en las coincidencias observadas sobre el conjunto de los dos primeros, resolviéndose las dudas siempre en función de las especies dominantes. En cuanto al cálculo de la afinidad, se prefirió aplicar un índice cualitativo ampliamente utilizado (Czechanovski) frente a las opciones cuantitativas, por entender que estas últimas presentan un alto grado de redundancia respecto a un método más potente como es el análisis de correspondencias. El algoritmo elegido para obtener los diagramas de afinidades o dendrogramas, ha sido el método UPGMA, por sus características de ser jerárquico, aglomerativo y «no pesado», lo que implica que se acumulen menos distorsiones en los sucesivos pasos respecto a los datos de partida (JONGMAN et al., 1987). Una vez establecidas las principales agrupaciones, se intenta poner de manifiesto algunos aspectos estructurales mediante la aplicación del índice $\mathrm{H}^{\prime}$ de Shannon-Weaver y de la expresión de heterogeneidad $\mathrm{h}=\mathrm{H}$ 'y $-\mathrm{H}^{\prime} \mathrm{a}$ (Margalef, 1968).

\section{RESULTADOS}

La ordenación de los inventarios en el plano principal (fig. 2), permite apreciar dos tendencias generales. La primera de ellas sigue aproximadamente la dirección del eje I; a partir de un comienzo algo confuso donde se superponen, al menos en parte, las comunidades cacuminales, se aprecia una seriación del arroyo en zona alta, media, media-baja y baja. El cauce, pedregoso y muy 
uniforme, no parece constituir motivo de diferenciación, pero sí la sombra de los bosquetes dispersos de robles, abedules, fresnos, alisos y avellanos, que aparecen en las zonas media-baja y baja. $\mathrm{Su}$ presencia lleva a que se separe un conjunto de comunidades (ombrófilas) del grupo con niveles más altos de insolación.

Otros dos aspectos se traducen en la formación de pequenas agrupaciones. Por una parte, las cinco comunidades más próximas al núcleo de población atravesado por el arroyo (Navacarros) presentan un cierto grado de nitrofilia; como ocurre con las ombrófilas, aparecen disgregadas de la línea principal de variación sin alejarse de la zona a la que pertenecen (zona baja). Por otra parte, en la zona media se presentan algunos paredones rocosos, donde el arroyo discurre muy encajado; en este caso. el nivel de inundación está formado por enclaves rezumantes donde predominan las especies fisurícolas. Dichos enclaves quedan también diferenciados y cercanos a la zona que les es propia.

La segunda tendencia general viene marcada por las zonas más cumbreñas. Siguiendo el eje II, se distinguen cinco grupos muy netos (muestreo selectivo). En posición extrema aparecen las comunidades de pequenas pozas y charcas de agua oligotrofa, propias de la zona cacuminal, que quedan un tanto aisladas. Siguen los manantiales y arroyos de alta montaña recién nacidos, de corriente relativamente fuerte y nivel hasta cierto punto estable. A medida que disminuye la fuerza de la corriente, por lo común al alcanzarse rellanos, se disponen las comunidades sumergidas de

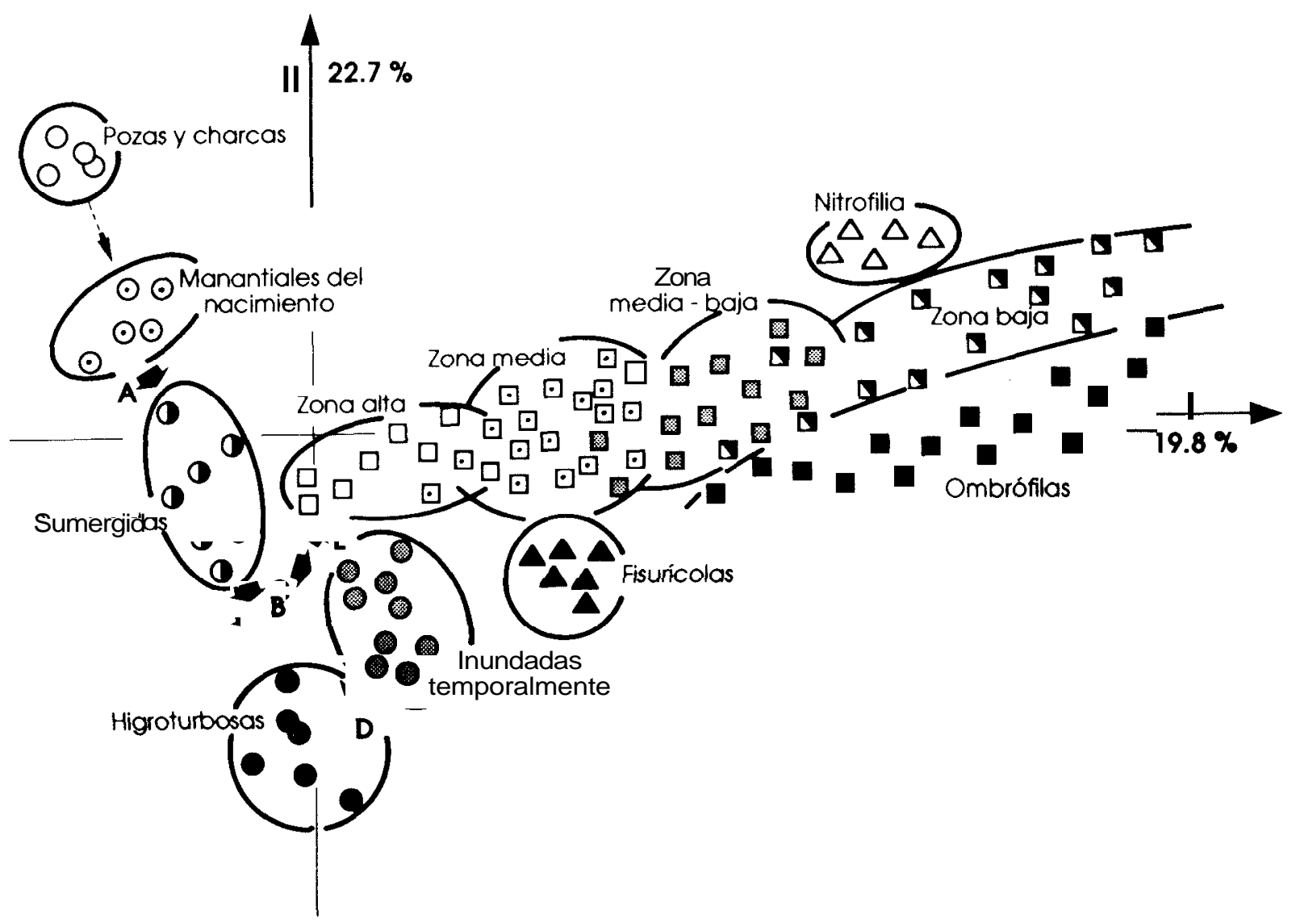

Figura 2.- Ordenación de las comunidades en el plano formado por los ejes I y II del análisis de correspondencias Ordering of communities on the planc formcd by axes I and II of the correspondence analysis. 
cubetas, pozas, charcos o regatos de agua meso-oligotrofa de nivel variable; cuando el agua comienza a divagar, y si no hay fuertes acumulaciones de fitomasa, aparecen las inundadas temporalmente (también de nivel variable), o bien las higroturbosas, cuando los depósitos de biomasa vegetal son fuertes. Por tanto, prescindiendo de las pozas y charcas de los lugares más altos, de carácter algo singular, el eje II puede interpretarse como un gradiente hacia estados remansados, a lo que se unen variaciones en la constancia del nivel del agua, acúmulo de biomasa, y cambio, en general, de condiciones oligotrofas a más eutrofas. Las flechas negras indican relaciones fitosociológicas entre grupos, como se pondrá de manifiesto en la discusión.

Si la diferenciación de las comunidades resulta evidente, aunque con algunas superposiciones, la de las especies aparece algo más compleja, debido a que determinados elementos pueden asociarse indistintamente a dos o varios grupos. No obstante, dada la caracterización mencionada por ejes que proporciona el análisis de correspondencias, su posición en el plano, y a la vista de la tabla de datos, grupos de comunidades y especies pueden relacionarse, con cierto grado de subjetividad, según se recoge en la tabla 1 , donde se indican, asimismo, las contribuciones relativas más importantes para los dos primeros ejes. En todo caso, el objetivo planteado no es describir la vegetación, sino establecer hasta qué punto es posible la tipificación de comunidades a partir de ella, si bien conviene hacer alguna matización sobre las que se van a denominar nitrófilas y fisurícolas. Las primeras, engloban una serie de especies de las que $\mathrm{U}$. dioica y $O$. crocata son claramente nitrófilas y buenas indicadoras de bordes de arroyos visitados por el ganado. También C. longus, aunque en grado inferior, es un claro ejemplo de este tipo de enclaves. En cuanto a C. pycnocephalus, V. officinalis, $S$. procumbens y L. perenne, en mayor o menor medida muestran tendencia hacia la nitrofilia, por lo que las comunidades en que son dominantes caben ser consideradas como nitrófilas, al menos en cierto grado. No obstante, sobre las características de las fitocenosis nitrófilas, particularmente en medios peculiares como el tratado, conviene referirse a Rivas Martínez (1977) y LADERO et al. (1982). Las especies fisurícolas y de paredones rocosos rezumantes no admiten ninguna duda en su diferenciación, como cabría esperar de lo contrastado del hábitat en que se desarrollan. Esto no quiere decir que su presencia sea siempre exclusiva de estas localizaciones, ya que, por ejemplo, P. vulgare y S. tenuifolium son también frecuentes en robledales. El resto, incluida la subfisurícola M. uniflora, están por lo común ligadas a la proximidad de los cursos de agua, en roquedos rezumantes y otros emplazamientos pedregosos húmedos. Lo restringido de la banda muestreada tal vez impone unos condicionantes que concretan demasiado el asentamiento de las especies, pero ello no significa la pérdida de validez en la tipificación.

Para el análisis de afinidad (datos cualitativos), se ha mantenido la división entre zonas cumbreñas y zonas de seriación longitudinal, eliminando las dos agrupaciones más desviadas de estas tendencias, es decir, aquellas a las que se acaba de hacer referencia: nitrófilas y fisurícolas (fig. 3). Respecto a los enclaves cacuminales (coeficiente de correlación cofenética de 0.502; p $<0,01)$, se observa que la separación de los distintos grupos es muy neta y los niveles de similitud intragrupales bastante elevados, si bien van disminuyendo paulatinamente desde pozas y charcas hasta las áreas inundadas temporalmente. En contraste, la similitud intergrupos se reduce mucho, particularmente para las pozas y charcas, de relaciones fitosociológicas menos marcadas con las restantes comunidades. La causa parece radicar de nuevo en la estratificación del muestreo, con ausencia de estadios intermedios.

La situación es distinta para la zonación (coeficiente de correlación cofenética de 0,364; $\mathrm{p}<0,01)$. Los grupos se yuxtaponen, y la variabilidad dentro de cada grupo es más elevada (afinidad relativamente reducida). Además, la unión entre grupos se realiza a niveles que no difieren mucho de algunas afinidades intragrupales. Todo ello es indicio de una considerable mezcla específica, si bien en la zona alta la similitud es aún notable, alcanzándose los valores más reducidos en las zonas media-baja y baja.

Algunos de estos aspectos pueden completarse mediante el análisis estructural basado en la diversidad y en la heterogeneidad (fig. 4). Para la zona cacuminal, la diversidad suele ser reducida, aunque aumenta desde las pozas y charcas hasta las comunidades inundadas temporalmente (en con- 
Tabla 1.- Especies que caracterizan a las comunidades diferenciadas mediante el análisis de correspondencias. También se indican las contribuciones relativas $(\geqslant 0,10)$ para los dos primeros ejes.

Species characterizing the communities differentiated by the correspondence analysis. The contributions relatives $(\geqslant 0.10)$ for the two firsts axes are also indicated.

Comunidades de pozas y charcas de la zona cacuminal

Isoetes velata L. (1: $0.36 ; 11: 0.54)$

Myosurus minimus L. (I: $0.32 ; 11: 0.58)$

Comunidades propias de manantiales y arroyos recién nacidos de alta montana

Montia fontaria L. (1: $0.22 ; 11: 0.31)$

Stellaria alsine Grimm (I: 0.15; 11: 0.23)

Saxifraga stellaris L. (1: 0.12 ; II: 0.18$)$

Sagina nevadensis Boiss. \& Reutcr (1: 0.30)

Mucizonia lagascae (Pau) Laínz (1: 0.21 )

Comunidades sumergidas de cubetas, pozas. charcas o regatos de agua meso-oligotrofa de nivel variable

Ranunculus flammula L. (1: 0.17)

Veronica scutellata L. (1: 0.15)

Comunidades higroturbosas

Carex nigra (L.) Reichard (II: 0.41)

Parnassia palustris L. (II: 0.32)

Drosera rotundifolia L. (11: 0.32)

Viola palustris L. (II: 0.28 )

Sedum villosum L. (11: 0.13)

Comunidades inundadas temporalmente

Alopecurus aequalis Sobol. (II: 0.16)

Scirpus setaceus L. (II: 0.11)

Juncus tenageia Ehrh. (II: 0.10)

Juncus buffonius L.

Comunidades de la zona alta Epilobium collinum C.C. Gmelin

Carex lepidocarpu Tausch

Veronica serpylifolia $\mathrm{L}$.

Comunidades de la zona media

Polygonum hydropiper I

Ranunculus ololeucos Lloyd

Geum urbanum L

Calliriche brutia Petagna

Carex muricata L.

Centaurea triumfetti All

Comunidades de la zona media-baja

Caltha palustris L (1:0.10)

Saxifraga granulata L

Epilobium tetragonum $\mathrm{L}$

Myosotis debilis Pomc

Eleocharis multicaulis (Sm.) Desv

Comunidades de la zona baja

Veronica beccabunga L. (1:0 16: 11:0 12)

Rumex bucephalophorw L. (1: 0.27)

Trifolium striatum L. (1: 0.25)

Prunella vulgaris L. (1: 0.23)

Lotus uliginosus Schkuhr (1: 0.20)

Trifolium repens L. (1: 0.18)

Juncus squarrosus L. (I: 0.10)

Nasturtium officinale $\mathrm{R}$. Br. (1: 0.10 )

Comunidades ombrófilas, por lo común en la zona baja o media-baja

Saponaria officinalis L. (I: 0.22)

Primula vulgaris Hudson (I: 0.18)

Solanum dulcamara L. (I: 0.16 )

Carex laevigata $\mathrm{Sm} .(1: 0.14)$

Salix fragilis L. (1: 0.12)

Lonicera periclymenum $\mathrm{L}$.
Juncus bulbosus L. (1: 0.10$)$

Ranunculus omiophyllus Ten

Agrostis canina L. (II: 0.11)

Carex echinata Murray

Juncw articulatus $\mathrm{L}$.

Juncus pygmaeus L.C.M. Rich.

Radiola linoides Roth

Hypericum humifusum $\mathrm{L}$.

Lythrum portula (L.) D.A. Webb

Spergularia capillacea (Kindb. \& Lange) Will

Angelica major Lag.

Deschampsia flexuosa (L.) Trin

Cirsium palustre (L.) Scop.

Polygonum lapathifolium $\mathrm{L}$

Ranunculus penicillatus (Dumort.) Bab

Epilobium montanum L.

Mentha pulegium L.

Succisa pratensis Moench

Musgos

Polygonum persicaria $\mathrm{L}$

Trifolium strictum L.

Apium repens (Jacq.) Lag.

Veronica anagallis-aquatica $\mathrm{L}$.

Gagea pratensis (Pers.) Dumort

Juncus acuiflorus Ehrh.

Wahlembergia hederacea (L.) Reich

Ranunculus paludosus Poiret

Lepidium heterophyllum Bentham

Trifolium duhium Sibth

Juncus effusus $\mathrm{L}$

Knautia dipsacifolia Kreutzer

Arhyrium filix-femina (L.) Roth

Galium broterianum Boiss. \& Reuter

Thalictrum speciosissimum $\mathrm{L}$

Luzula sylvatica (Hudson) Gaudin

Salix atrocinerea Brot

Rrachypodium sylvanicum (Hudson) Beauv 
Comunidades con un cierto grado de nitrofilia

Urtica dioicu L. (11: 0.11)

Oenanthe crocatu L.

Carduus pycnocephalus $\mathrm{L}$

Cyperus longus $L$.

Comunidades rezumantes rocosas y fisuricolas Polypodium vulgare L. (11: 0.12)

Sibthorpia europaea L. (11: 0.10)

Antirrhinum meonanthum Hoffmanns. \& Link
Sagina procumbens $L$

Verbena officinalis $L$.

Lolium perenne $L$.

Mimulus moschans Douglas

Melica uniflora Retz.

Sedum tenuifolium (Sibth. \& Sm.) Strobl gruencia con las variaciones de la afinidad). Influye el pequeño número de especies, pero también las acentuadas condiciones ecológicas, que potencian la dominancia. En la seriación longitudinal del arroyo la diversidad es más elevada, excepto para la zona alta, que participa, al menos en parte, de las mismas causas que se acaban de citar (cabría incluir también a las comunidades ombrófilas). La zona baja y, particularmente, la mediabaja alcanzan los valores más altos (FERNÁndez AlÁEZ et al., 1984); se trata del tramo de mayor relajamiento ambiental, ya que no está influenciado ni por la sombra ni por las condiciones, hasta cierto punto severas, del nacimiento del arroyo (comparar de nuevo con las variaciones de la afinidad). En cuanto a las comunidades nitrófilas y fisurícolas, la situación es distinta para unas y otras. La nitrofilia, dado que no es muy acusada, permite la entrada de varias especies no nitrófilas, y la diversidad es relativamente alta; los medios fisurícolas, por el contrario, son severos y muy selectivos, por lo que presentan la diversidad más baja del conjunto.

La heterogeneidad evoluciona de forma parecida a la diversidad, pero existen variantes de interés. Así, el valor máximo alcanzado por las comunidades ombrófilas podría deberse a la variedad de árboles de ribera y a la distinta potencia de los bosquetes. Las comunidades nitrófilas son poco heterogéneas, dado que siempre destacan los mismos elementos, aunque las especies acompañantes puedan diferir bastante. Por el contrario, en las fisuras de las rocas casi siempre dominan unas pocas especies, pero la falta de constancia hace que aumente comparativamente la heterogeneidad que, con todo, es baja, debido al escaso número de especies. La mínima heterogeneidad se relaciona con las pozas y charcas, enclaves de composición florística siempre muy parecida.

\section{DISCUSIÓN Y CONCLUSIONES}

Siguiendo la figura 2, en la Lona más elevada las comunidades de pozas y charcas aparecen con un cierto aislamiento, aunque pueden relacionarse (flecha a trazos) con la vegetación helofítica de pequeña talla propia de manantiales y arroyos recién nacidos del piso montano u oromediterráneo. Dicha vegetación se desarrolla en aguas de temperatura y composición química bastante constante durante todo el ano y constituye, dentro de la clase Montio-Cardaminetea Br. Bl. y R. Tx. 1943 el orden Montio-Cardaminetaliu Pawlowsky 1928 em. Maas 1959. Su continuidad más directa (flecha A) puede encontrarse en las comunidades sumergidas de cubetas, pozas, charcas o regatos, ya dentro de la clase Littorelletea Br. Bl. y R. Tx. 1943 y del orden Littorelletalia W. Koch 1926. A partir de aquí, existen dos posibilidades. Una de ellas es el paso (flecha B) a las comunidades higroturbosas de la clase Scheuzerio-Curiceteu (fuscae) nigrue (Nordhagen 1936) em. R. Tx. 1937, y del orden Caricetalia (fuscae) nigrae $W$. Koch 1926, propio de medios ácidos y de clima frío, que se desarrolla entre 1800 y $2200 \mathrm{~m}$ de altitud ( $\mathrm{RI}_{\mathrm{I}}$ vas Martinez, 1963). La otra posibilidad es la transición (flecha $\mathrm{C}$ ) a las comunidades inundadas temporalmente de la clase Isoeto-Nanojuncetea Br. Bl. y R. Tx. 1943, y del orden Isoetalia Br. Bl. 1931, comunes en pozas o cubetas graníticas por encima de los 1500-1600 m. Ocupan áreas muy pequeñas de suelo ralo $\mathrm{y}$, fitosociológicamente, 
contactan con las de Littorelletulia, anteriormente reseñadas. Cuando se inicia la colmatación por encenagamiento (flecha D), se originan las formaciones higroturbosas, presididas por Curex nigra (Herrero, 1985).

El contacto entre esta serie de variación y la de zonación longitudinal se ha establecido, de forma un tanto arbitraria, entre las comunidades sumergidas e inundadas (flecha E). Dicha zonación longitudinal resulta muy clara (al contrario de lo que ocurre en algunos trabajos sobre arroyos de montaña), posiblemente tanto por la uniformidad del sustrato duro del Arroyo del Oso, lo que impide distorsiones debidas a la alternancia de medios, como por no haberse separado las especies sumergidas de las emergidas (si bien sometidas a inun- daciones temporales). La fuerza de la corriente decae de manera paulatina, aunque no pronunciada, a medida que el arroyo se ensancha, y aquí pudiera encontrarse una tercera razón para que se detecten zonas bien definidas.

Con todo, la peculiaridad de algunos hábitats impide obtener una secuencia completamente lineal. Así, las paredes graníticas ocasionales dan lugar a la presencia de especies fisurícolas y alguna subfisurícola. Igual cabe decir respecto a las comunidades nitrófilas, fitocenosis limitadas a las cercanías del núcleo urbano de Navacarros, pero que se encuentran en plena expansión dados los aportes cada vez más intensos de materia orgánica. Si de los dos factores de incidencia tradicional el sustrato carece de repercusiones aparentes en

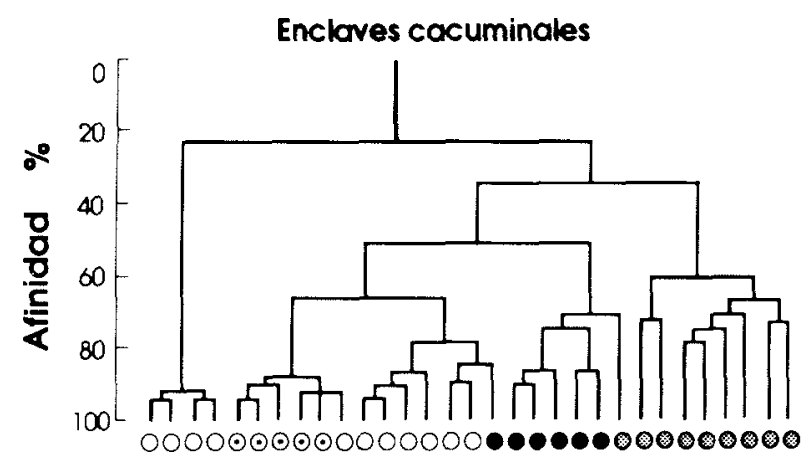

Zonaciön longitudinal

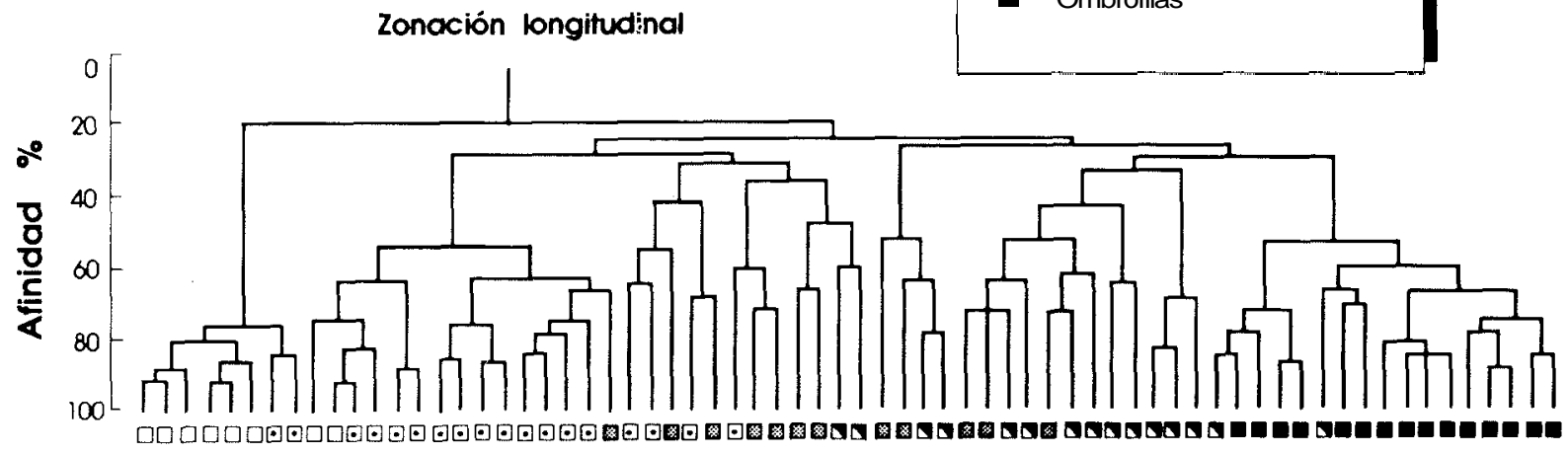

Figura 3.- Análisis de clasificación. Se consideran por separado los enclaves cacuminales y la zonación longitudinal

\footnotetext{
Classification analysis. The cacuminal enclaves and the longitudinal zonation are considered separatelv.
}
- Pozas y charcas
○ Manantiales del nacimsnto
O Sumergidas
- Higroturbosas
- Inundadas temporalmente

$\square \quad$ Zona alta

$\square \quad$ Zona media

: Zona media - baja

$\square \quad$ Zona baja

- Ombrófilas 
este caso, ocurre todo lo contrario con el efecto de la sombra. Se ha discutido si a dicho efecto no contribuiría también el de los árboles sobre las características del agua de infiltración, pero el hecho de que los puntos de muestreo sombreados por estrechos bosques de galería (agua de infiltración poco alterada) aparezcan entremezclados con otros lugares más boscosos, indica que en la distribución de la vegetación interviene muy probablemente el sombreado y no algún otro efecto (AMOR, 1984).

Dado que la distribución de las plantas acuáticas está relacionada con la cantidad y calidad de radiación visible que filtra el dosel de árboles y arbustos riparios (FAssett, 1957; Westlake, 1966; Haslam, 1978), la orientación de los cauces y la pendiente pueden interferir en las diferenciaciones esperadas. Por otra parte, la especie arbórea carece de influencia, o bien ésta queda enmascarada por las variaciones de densidad. Únicamente en el caso de los alisos se produce un descenso considerable de la vegetación de los cauces, circunstancia que ha sido constatada por BERMúdez de CAstro et al. (1981).

La ordenación que se consigue mediante el análisis de correspondencias podría deberse a la dominancia ejercida por algunas especies. Sin embargo, mediante el análisis cualitativo de afinidad se aprecia que también las especies en sí son decisivas para conseguir segregaciones de gran precisión en los enclaves cacuminales, pero a costa de no haber realizado el muestreo al azar (falta

\section{HETEROGENEIDAD}

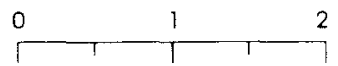

Pozas y charcas

Manantiales del nocimiento

Sumergidas

Higroturbosas

Inundadas temporalmente

Zona medio

Zona media - boja

Zona boja

Ombrófilos

Nitrofilio

Fisuricolas
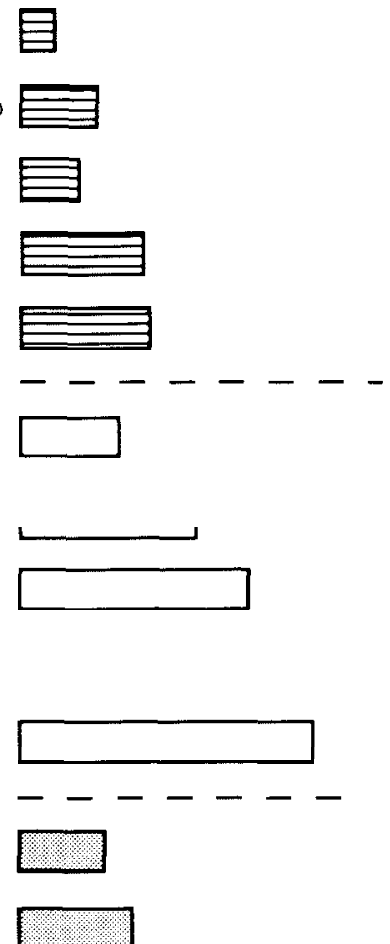

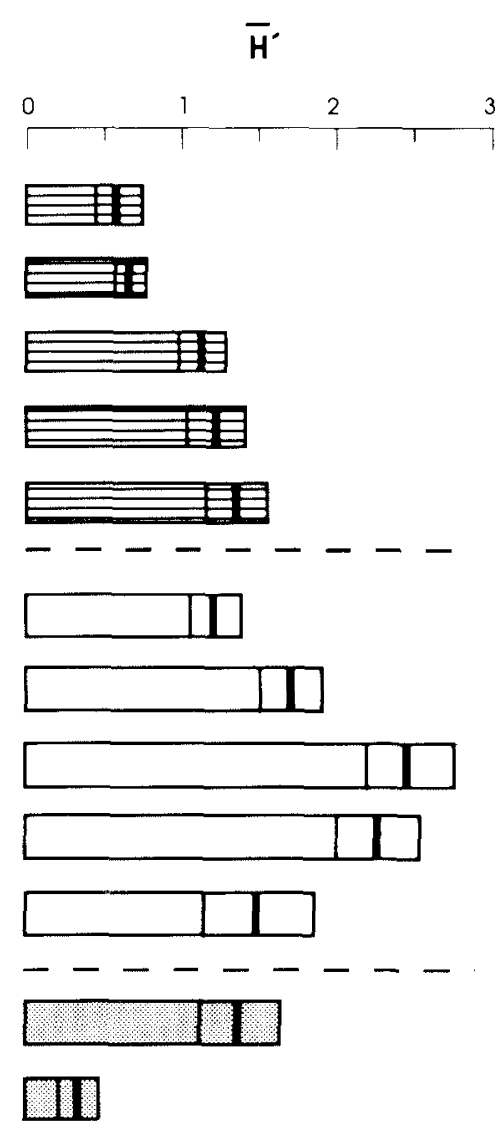

Figura 4.- Diversidad media (se indican los intervalos de confianza para $\mathrm{p}<0.05$ ) y heterogeneidad de las comunidades diferenciadas mediante el análisis de correspondencias. Se conserva el orden de los sectores y grupos definidos mediante dicho análisis. Mean diversity (confidence intervals for $\mathrm{p}<0.05$ are indicated) and heterogeneity of the communitics differentiated by the correspondence analysis. The order of ths sectors and groups defined by this analysis is preserved. 
de estados de transición). No ocurre lo mismo con la zonación longitudinal. Excepto para el tramo alto, cuando se pasa al nivel cualitativo los inventario aparecen bastante entremezclados, al ser varias las especies comunes o indiferentes que admiten amplios recorridos a lo largo del cauce, aunque sólo lleguen a predominar en puntos concretos. Es probable incluso que se produzca una considerable disparidad de resultados entre tratamientos cuantitativos y cualitativos, sobre todo en arroyos más cortos, por lo que nada tiene de extraño que iluminación y sustrato, los factores donde las coincidencias son mayores, se hayan convertido en los agentes causales preferentemente tratados.

La diversidad alcanzada por cada grupo viene a ser coincidente con los niveles de afinidad, lo que es una nueva garantía de la partición zonal, aunque ésta quede amortiguada en el tratamiento cualitativo. Las discrepancias son fácilmente interpretables en función del número de especies o de las relaciones de dominancia. Así, para la nitrofilia, el elevado número de especies acompañantes proporciona una diversidad alta, mientras que la gran incidencia de las especies auténticamente nitrófilas da lugar a una heterogeneidad baja; la acusada heterogeneidad de las comunidades ombrófilas puede estar ligada en pequeña parte a la especie leñosa que causa el sombreado, pero sobre todo a las diferencias en la densidad de los bosquetes, que no proporcionan igual intensidad de luz, y a su pertenencia a dos zonas, la media-baja y la baja, de características algo dispares. aunque el papel sobresaliente de la sombra no permita establecer distinciones. Hay que destacar. por tanto, cómo ante la imposición de una causa de fuerza mayor la zonación se desvanece, hecho que posiblemente ocurre en otros casos, donde las condiciones alternantes de luz se proyectan a lo largo de todo el cauce. Las características locales parecen desempeñar un papel fundamental frente al intento de buscar tendencias generalizables; es de esperar que la suma de aspectos puntuales permita al menos establecer un esquema en el que basar futuras actuaciones.

\section{Bibliografia}

AMOr. A.. 1984. Distribución de la vegetación macrofitica en arroyos y gargantas de la cuenca alta del rio Tormes. Tesis de Licenciiiturn, Universidad de Salamanca. $129 \mathrm{pp}$

Bermúdez de Castro. F.. P. Barbiko \& M.G. Martín. 1981. Doseles de alisos y crecimicnto de plantas acuáticas Actas del I Congreso Español de Limnologia, Barcelona: 167-171.

FASSETT. N.C., 1957. A mamual of aquatic plants. University of Wisconsin Press. Madison. $210 \mathrm{pp}$.

Fi rnandez Alaiz., M.C., E. Luis \& M. Firnandi 7 Aiatz. 1984. Estudio de la composición y distribución de la vegetación ribereña en la cuenca alta del río Bernesga, León. Limnética. 1 (1): 159-168.

García Romriguez, A.. 1964. Los suelos de la provincia de Salamanca. Publicaciones del IOATO. Salamanca. 145 pp.

Garmendia. S., 1964. Estudio climatológico de la proviriciu tic. Salamanca. Publicaciones del IOATO, Salamanca. $229 \mathrm{pp}$.

Haslam. S.M., 1978. River plants. The macrophytic vegetation of water-courses. Cambridge University Press. $396 \mathrm{pp}$.

Herrero, F., 1985. Flórula y vegetación de los términos municipales de Navacarros y La Hoya. Tesis de Licenciatura. Universidad de Salamanca. $151 \mathrm{pp}$.

Horr. M., 1978. La végétation des euiix corantes. In: Les eaux douces d'Alsace: 28-44. Mars et Mercure, Wettolsheim.

HOLMES. N.T.H., 1983. 'l'yping British rivers according to their flora. Focus on nature conservation. N." 4 Nature. Conservancy Council.

JONGMAN, R.H.G.. C.S.F. ter BKAAK \& O.F.R. van TONGEREN, 1987. Data analysis in community and landscape ecology. Pudoc. Wageningen. 299 pp.

Ladero. M.. F. Navarro \& C.J. Valll, 1983. Comunidades nitrófilas salmantinas. Studia Botánica, 2: 7-67.

Margalef, R., 1968. Perspectives in ecological theory. University of Chicago Press. $111 \mathrm{pp}$.

Margalei, R., 1983. Limnología. Ed. Omega. Barcelona. $1010 \mathrm{pp}$.

Rivas Martínez, S., 1963. Estudio de la vegetación y flora de la Sierra de Guadarrama y Gredos. Ann. Inst. Bot. A.J. Cavanilles, 21: 1-325.

Rivas Martínez. S., 1975. Datos ecológicos sobre la vegetación acuática continental. Ann. Inst. Bol. A.J. Cavanilles. 32: 199-205.

Rivas Martínez, S.. 1977. Datos sobre la vegetación nitrófila española. Acta Bot. Malacitana, 3: 159-167.

WESTLAKE, D.F.. 1966. The light climate for plants in rivers. In: Light as an ecological factor: 99-119. Blackwell, Oxford. $452 \mathrm{pp}$.

WeTZEL, R.G., 1975. Primary production. In: River ecology (Whitton. B.A. ed.): 230-247. Blackwell, Oxford. 725 pp.

Whitton, B.A., 1975. Preface. In: Whitton. B.A. (ed.), River ecology: IX-X. Blackwell. Oxford. $725 \mathrm{pp}$. 